\section{EL ROL DE LAS FUERZAS ARMADAS EN EL CICLO DE GESTIÓN DEL RIESGO DE DESASTRES EN CHILE: UNA RELACIÓN EN DESARROLLO}

Gabriel E. Cabezas Ríos ${ }^{{ }^{*}}$

\section{RESUMEN}

El ciclo de gestión del riesgo de desastre va más allá de posicionar a los diferentes actores en cada una de sus fases. Dado que a la fecha de esta publicación aún se encuentra en el Congreso Nacional de Chile el proyecto de ley que transformará el Sistema Nacional de Protección Civil en Chile. Este artículo reflexiona sobre algunos de sus fundamentos y sobre el nuevo rol de las Fuerzas Armadas dentro de aquel ciclo. Después del terremoto ocurrido el 27 de febrero de 2010 en el Maule, Chile, la participación de las Fuerzas Armadas en diferentes escenarios de desastre y emergencia, tanto dentro como fuera de Chile, ha sido significativa. A través del análisis documental y entrevistas en profundidad, este trabajo entrega una mirada empírica de la relación en desarrollo entre las Fuerzas Armadas y el Sistema Nacional de Protección Civil en Chile. Algunas de las observaciones apuntan a que los medios y las capacidades de despliegue de las Fuerzas Armadas han contribuido a que los sistemas de respuestas ante desastres sean más oportunos y eficaces. No obstante, la relación mencionada requiere de más investigaciones y reflexión por parte de todos los actores involucrados, en especial por sus implicaciones sociales.

\section{PALABRAS CLAVE}

Ciclo de gestión del riesgo de desastre, Fuerzas Armadas, Protección Civil, Desastres, Emergencias

\section{THE ROLE OF THE ARMED FORCES IN THE CYCLE OF DISASTER RISK MANAGEMENT IN CHILE: A DEVELOPING RELATIONSHIP}

\section{ABSTRACT}

The disaster risk management cycle is more than the sum of its phases and actors. This article aims to reflect on some of the cycle's grounds and especially on the role of the Armed Forces during response. This is relevant today because of the bill that will transform the National Civil Protection System in Chile is still being discussed in the Chilean National Congress. After the earthquake that occurred on February 27, 2010 in Maule, Chile, the participation of the Armed Forces in different disaster and emergency scenarios, both nationally and internationally, has been significative. Through documentary analysis and in-depth interviews, this work provides an empirical look at the developing relationship between the Armed Forces and the National Civil Protection System in Chile. Some observations point out that the capacities and resources -human and on logistics- of the Armed Forces have contributed in making disaster responses timely effective. Nevertheless, the mentioned relationship needs to be further scrutinized by all actors considering its social implications.

\section{KEYWORDS}

Disaster risk management cycle, Armed Forces, Civil Protection, Disasters, Emergencies
1. Universidad de Valparaíso, Valparaíso, Chile.

*Autor de correspondencia: gabrielcabezasr@gmail.com

\section{RECIBIDO}

13 de octubre de 2019

\section{ACEPTADO}

5 de febrero de 2020

\section{PUBLICADO}

1 de julio de 2020

\section{Formato cita}

Recomendada (APA): Cabezas Ríos, G.E. (2020). El Rol de las Fuerzas Armadas en el Ciclo de Gestión del Riesgo de Desastres en Chile: Una relación en desarrollo. Revista de Estudios Latinoamericanos sobre Reducción del Riesgo de Desastres REDER, 4(2), 111-122.

\section{(ब) $(\Phi \otimes$}

Todos los artículos publicados en REDER siguen una política de Acceso Abierto y se respaldan en una Licencia CreativeCommons Atribución-NoComercial 4.0 Internacional.

Revista de Estudios Latinoamericanos sobre Reducción del Riesgo de Desastres (REDER)

Diseño: Lupe Bezzina Tipografía: Hospital 


\section{INTRODUCCIÓN}

La diversa geografía del territorio chileno ha contribuido a que las emergencias y los desastres sean variados, desde las inundaciones en la desértica zona norte hasta el 'terremoto blanco' que ha afectado la región de Magallanes. Sin olvidar la extensa costa del Pacífico por el oeste y el cordón volcánico de los Andes por el este, acentuando la exposición de la población. Y si a ello consideramos los otros "factores subyacentes" (ONU, 2015, p.10), presenta un escenario complicado a la hora de evaluar los riesgos. Variabilidades que han desencadenado eventos destructivos a lo largo del territorio, comprometiendo los recursos fiscales, gasto público que debe ser empleado para reaccionar en la fase de respuesta y posteriormente concretar procesos de reconstrucción frente a imprevistos económicos. Un ejemplo de ello es lo que manifiesta la Organización para la Cooperación y el Desarrollo Económico (OCDE), al enunciar una lista que comprende las pérdidas económicas producidas por los desastres entre los países miembros del G20, donde Chile la encabeza (CNID, 2016). Dentro del contexto, cabe mencionar lo expuesto por el Concejo Nacional de Innovación para el Desarrollo (CNID), que enuncia los eventos sísmicos cuya escala de magnitud $\left(\mathrm{M}_{\mathrm{w}}\right)$ sobrepasaron los 8.0 durante los años 1960, 2010, 2014 y 2015 (р.20), siendo el primero el de mayor magnitud en la historia de los terremotos medidos con instrumentos modernos. Paralelamente, no se deben dejar de lado los eventos volcánicos, los que presentan un riesgo latente a lo largo del territorio nacional con un importante nivel de actividad (SERNAGEOMIN, 2020). Por lo anterior, la resiliencia económica y la gobernanza son fundamentales a la hora de fortalecer la Gestión del Riesgo de Desastres (GRD).

En relación a lo expuesto es preciso tener en cuenta que la magnitud y frecuencia de los eventos extremos han ido en aumento, especialmente los climáticos, lo que, sumado al crecimiento descontrolado de las ciudades y los asentamientos no regulados, ha aumentado la exposición de la población. Tal exposición no solo aumenta las condiciones de vulnerabilidad ante los efectos de un evento destructivo si no también, el compromiso económico que debe desarrollar la autoridad gubernamental para enfrentar las variables y el nivel de riesgo al que la población esta expuesta.

Por otra parte, la experiencia nacional ante las consecuencias de los eventos destructivos han hecho que las autoridades desarrollen políticas para prevenir y mitigar los riesgos, motivando el fortalecimiento del Sistema Nacional de Gestión del Riesgo de Desastres (SNGRD), sobre todo lo concerniente a la fase de respuesta donde convergen diversas instituciones y cuyo objetivo se centra en restaurar en el menor tiempo posible los servicios básicos de la población afectada y de recuperar el funcionamiento de la infraestructura sensible como los centros de atención médica de urgencias, los de control de pacientes crónicos y electrodependientes, entre otras instalaciones necesarias para la administración del gobierno comunal, regional o nacional.

Lo anterior, precisa de una institucionalidad fuerte ante las probabilidades descritas y de la complejidad del proceso de reacción de los sistemas de emergencias, requiriendo que la Oficina Nacional de Emergencia del Ministerio del Interior y Seguridad Pública (ONEMI) sea una organización con la capacidad suficiente para enfrentar los nuevos escenarios que van en directa relación con el desarrollo de la urbanización ya sea ésta regulada o no. Pero hoy en día, la cober tura de reacción solo queda circunscrita a la coordinación de los medios de emergencia respectivos y no a la disposición directa de ellos, motivo por el cual los Comités de Operaciones de Emergencia (COE) cumplen un rol fundamental a la hora llevar a cabo las coordinaciones necesarias entre las diferentes instituciones públicas y privadas que intervienen en las emergencias o desastres (BCN, 2017). Instituciones que a nivel comunal poseen capacidades muy limitadas y su actuar va en directa relación con los recursos disponibles. Es por ello la importancia de fortalecer el actual Sistema Nacional de Protección Civil (SNPC), que sea un mecanismo oportuno y eficaz en la respuesta y eficiente en la reconstrucción. Pero es en la primera de ellas donde se requiere un gran despliegue de medios y recurso humano que en ocasiones no pueden ser proporcionados necesariamente por la comuna o éstas no cuentan con las capacidades suficientes para responder oportunamente a las necesidades inmediatas de la población afectada, requiriendo necesariamente de un apoyo externo. En este contexto se consideran a las Fuerzas Armadas, que en tales circunstancias son instituciones que se encuentran presente en todo el país a través de sus respectivas unidades y cuya acción para actuar se encuentra ajustada a un tiempo prudente de respuesta y que complementadas a los gobiernos locales pueden aumentar las capacidades de despliegue del SNPC. 


\section{METODOLOGÍA}

La metodología empleada en el presente trabajo fue estructurada en base a la información obtenida de la revisión bibliográfica y documental de la Defensa y de las principales organizaciones del Estado chileno que intervienen en el SNPC, además del conocimiento empírico y experiencias obtenidas de las fuentes primarias, información derivada de entrevistas semi-estructuradas realizadas a funcionarios responsables de la conducción militar una vez declarado el 'Estado de Excepción Constitucional' en enero del año 2017, debido al incendio que afectó a Valparaíso, Chile y de la experiencia del autor que en base a la observación directa graficada en relatos específicos de diversos eventos como: el terremoto de Haití ocurrido en enero del año 2010 mientras se encontraba desplegado en calidad de enfermero en la Misión de Estabilización de las Naciones Unidas en Haití (MINUSTAH), en la emergencia que comprometió a los 33 mineros de la Mina San José de Copiapó, Chile apoyando en el proceso de capacitación al equipo médico desplegado, en el apoyo brindado en el desastre ocurrido en la mina La Preciosa, Colombia, cuya colaboración se centró en el proceso de investigación de la tragedia y, la participación en el equipo médico compuesto por profesionales civiles y militares que se desplegó en el cerro Las Cañas de Valparaíso debido al gran incendio ocurrido en marzo del año 2014.

Por lo tanto y de acuerdo a lo expresado, se evaluó la información histórica y documental, desarrollando así un contenido que permita ilustrar el rol de las Fuerzas Armadas dentro del actual SNPC en las diferentes fases del Ciclo de la Gestión del Riesgo de Desastre (CGRD), considerando que dadas las diferentes situaciones acaecidas en Chile han requerido una presencia más activa de la Defensa en los respectivos Comités de Protección Civil (CPC) y en los COE (BCN, 2011) ya sean estos comunales, regionales o nacional.

\section{CHILE, SU POSICIONAMIENTO Y PARTICIPACIÓN EXTERIOR}

Para dimensionar el rol que tiene Chile en materia del estudio de la Gestión del Riesgo de Desastres (GRD) en la región y globalmente, es necesario hacer algunas menciones de su participación recurrente. A la fecha del presente texto la última participación de Chile a nivel global se llevó a cabo en la "VI Plataforma Global para la Reducción del Riesgo de Desastres" realizada en la ciudad de Ginebra, Suiza, en mayo de 2019 (UNISDR, 2019), donde se expresa que el país debido a su vulnerabilidad y a las consecuencias destructivas de las emergencias y los desastres ha comprometido 'pérdidas anuales en las últimas tres décadas cercanas al 1,2\% de su PIB' (ONEMI, 2019), siendo un claro ejemplo de lo perjudicial que puede llegar a ser para el sistema económico un evento destructivo e influir en el desarrollo del país.

Regionalmente, las actividades en materia de GRD son variadas y van desde reuniones de alto nivel ejercidas por las autoridades de gobierno respectivas, hasta trabajos o ejercicios militares conjuntos, reforzando de esta forma las capacidades de interacción entre las naciones ante las consecuencias de un desastre. En este contexto se puntualiza la Conferencia de Ministros de Defensa de Las Américas (CMDA, 2018a), organización multilateral presidida actualmente por Chile y que integra a las autoridades de la Defensa del hemisferio.

En relación a lo expuesto, durante el año 2018 se desarrollaron diversas reuniones donde grupos de trabajos (CMDA, 2018b) elaboraron informes atingentes a la participación de las Fuerzas Armadas en el CGRD, entre ellos se mencionan los siguientes:

- La Política de cooperación en defensa y seguridad hemisférica: Asistencia humanitaria en emergencias,

- La Participación de las Fuerzas de Defensa y Seguridad con respecto a la Protección Ambiental y la resiliencia climática.

- La evolución del papel de las Fuerzas Armadas en materia de Defensa y Seguridad; y Mecanismos de cooperación y coordinación en los temas de Búsqueda y Rescate.

Cabe mencionar que los grupos de trabajos señalados desarrollaron una serie de recomendaciones enfocadas a la integración de la Defensa en la GRD, siendo algunas de ellas:

- Mejorar las coordinaciones entre los actores involucrados en el CGRD.

- El trabajo conjunto y de cooperación mutua entre los países miembros.

- 'La capacitación en materia de respuesta a desastres'.

- Desarrollar estrategias para 'adaptarse a los impactos del cambio climático', entre otras. 
Puntos que conducen al fortalecimiento de las instituciones de la Defensa y del propio SNGRD para salvaguardar y garantizar la seguridad de la población ante las consecuencias de un evento destructivo.

Otra de las actividades a nivel regional fue la IV Reunión de Ministros de Defensa del Pacífico Sur (MDN, 2019a), organización multilateral que promueve la cooperación entre países miembros, siendo unos de los temas: la asistencia y ayuda humanitaria ante desastres, circunstancia en que el gobierno de Chile establece un acuerdo con Fiji y Australia para trabajar en forma conjunta.

Por otra parte, cabe destacar dos de los diferentes ejercicios multinacionales o binacionales realizados en Chile y sustentados en el empleo de las Fuerzas Armadas que se integran en la 'fase de respuesta', siendo uno de ellos el ejercicio multinacional Parthnership of the Americas (Embajada de los EEUU en Chile, 2014) ejercicio militar conjunto que contó con la participación de unidades militares de ocho países desarrollado en la comuna de Pichidangui, Chile, y cuyo objetivo fue dar apoyo humanitario a la población afectada ante las consecuencias generadas por un terremoto simulado de magnitud $8.8\left(\mathrm{M}_{\mathrm{w}}\right)$. Capacidades que, independiente de las condiciones geográficas y la estructura de mando y control, coordinados lograron una respuesta oportuna y eficaz, por lo tanto, las experiencias y el conocimiento adquirido durante el simulacro fortalecen el trabajo conjunto de las unidades, del mando operativo y de las diferentes autoridades presentes en el ejercicio.

Otro simulacro que cabe destacar, es el ejercicio militar binacional Solidaridad que se realiza en conjunto con la República de Argentina y surge de un acuerdo suscrito en el año 1997, estableciéndose el Decreto 254 Acuerdo con Argentina sobre cooperación en materia de catástrofes (BCN, 2002), cuyo objetivo se centra en el entrenamiento combinado de las Fuerzas Armadas para fortalecer el equipo de trabajo binacional ante las consecuencias de un desastre, mejorando las estrategias que se desarrollan en todo el CGRD.

Lo expresado es un claro ejemplo de la dinámica de las diferentes autoridades de gobierno por mejorar la participación, las condiciones, el conocimiento, la experiencia de la Defensa en la GRD tanto nacional como continentalmente y la representación gubernamental en el contexto global, cuyo objetivo se centra en reducir el Riesgo de Desastres (RD) y por consiguiente fortalecer aún más la gobernanza y la institucionalidad nacional del futuro Sistema Nacional de Gestión del Riesgo de Desastres.

\section{LA NUEVA INSTITUCIONALIDAD}

La historia sísmica de Chile, la variabilidad climática y los aspectos demográficos irregulares, son elementos que han presentado un mayor nivel de complejidad en relación al riesgo. Razones suficientes que han llevado a los gobiernos nacionales a replantear la ONEMI y así fortalecer el SNPC a través de una nueva organización que actualmente se encuentra en trámite en el Congreso Nacional (Senado de Chile, 2019). Lo anterior sumado a las sugerencias derivadas del Marco de Acción de Hyogo (MAH), a las experiencias del terremoto ocurrido en la región del Maule, Chile el 27 de febrero del año 2010 (desde ahora llamado 27F), a las emergencias estacionales, entre otras que requieren de una institucionalidad capaz de responder descentralizada y adecuadamente a lo largo del territorio nacional.

Dentro del proceso de reestructuración, la ONEMI consolida la Plataforma Nacional para la Reducción del Riesgo de Desastres, y ya el 2014 se establece la Política Nacional para la Gestión del Riesgo de Desastres. Posteriormente, se incorpora el Plan Estratégico 2019-2023 (ONEMI, 2018), iniciativas que en su conjunto han permitido avanzar en la modernización de la mencionada institución promoviendo un proceso de planificación que ayude a mejorar la gestión y las acciones estratégicas.

\section{LAS FUERZAS ARMADAS EN EL CICLO DEL RIESGO DE DESASTRES}

Constitucionalmente, uno de los deberes del Estado en Chile es "resguardar la seguridad nacional, dar protección a la población y a la familia [...]" (BCN, 2015, p.1), instrumentalización realizada a través del SNGRD, junto a la integración de la Defensa de acuerdo a las necesidades de apoyo que el gobierno central determine, dependiendo directamente de la magnitud del evento destructivo y de las capacidades de los gobiernos locales para responder a la emergencia que los afecta.

Se debe tener presente que las Fuerzas Armadas tienen un rol principal establecido por las figuras legales, siendo uno de ellos lo dispuesto por la Constitución chilena en base a lo siguiente: "Existen para la defensa de la Patria y son esenciales para la seguridad nacional" (p.47), además 
de lo determinado en el Estatuto Orgánico del Ministerio de Defensa Nacional que establece, entre otras cosas, su organización (BCN, 2010).

Para contextualizar en lo relativo a la seguridad y defensa, las Fuerzas Armadas como órgano del Estado tienen el deber de cumplir con lo establecido en la Constitución y las normas legales, siendo el objetivo la seguridad de la nación manteniendo la integridad de su soberanía, por ende, relacionadas directamente a los escenarios bajo condiciones bélicas. Pero hoy en día y debido a los riesgos descritos previamente, cobra relevancia un segundo rol de la Defensa que contempla el apoyo al SNPC ante las consecuencias de un evento destructivo catalogadas como emergencia o desastre o, bien podría ser consignado como acciones no bélicas de seguridad interior dirigidas a fortalecer las acciones del SNPC.

Lo anterior, no es posible sin la conducción estratégica (EMCO, 2014) que hace converger a las tres ramas de la Defensa según como lo determine la autoridad política respectiva, condición permanente en el empleo de las fuerzas que se despliegan en una zona afectada por una emergencia o desastre, las que debido a geografía chilena pueden diferir unas de otras y las necesidades serán de acuerdo al requerimiento de apoyo terrestre, aéreo o marítimo.

Antes de continuar, es necesario aclarar que las siguientes menciones del empleo de las Fuerzas Armadas antes del año 2010 serán de enfoque general debido a que no es el objetivo central de este estudio desarrollar un perfil histórico de larga data, si no, mencionar en forma breve su integración en materias de GRD que se consolidó después de las consecuencias acaecidas el $27 \mathrm{~F}$. En ese entonces y previo a la fecha enunciada la primera decisión no era disponer el despliegue de las ramas de la Defensa para consolidar el resguardo del orden público ante las consecuencias de un determinado evento destructivo, situaciones donde las acciones se acotaban a un objetivo específico y, por ende, aisladas. Participación que, por expresarlo de un modo, era limitada y no se evidenciaba claramente una estructura o interacción cívico-militar dinámica en todas las fases y etapas del CGRD. Esto deriva en que las acciones desarrolladas en la fase de respuesta, si bien en su tiempo se realizaban de la mejor forma, no se apreciaba una coordinación conjunta como la de hoy en día, siendo un ejemplo de los cambios el uso escalonado de los medios y recursos para apoyar en una determinada emergencia (ONEMI, 2002). Medidas locales que, si llegan a ser sobrepasadas $y$, considerando lo que disponga la autoridad de gobierno, podrían dar paso a que el presidente de la nación declare un 'Estado de Excepción Constitucional' para una jurisdicción afectada por un evento destructivo. La conducción y responsabilidad en estas circunstancias recae en la figura de una autoridad militar denominada 'Jefe de la Defensa Nacional' (BCN, 2015).

De acuerdo a lo expresado, es preciso deducir que el punto de inflexión del empleo de las Fuerzas Armadas ante las consecuencias de un evento de alta magnitud que obligó al gobierno de ese entonces a declarar Estado de Excepción Constitucional fue precisamente el 27F, decreto que consignó la presencia de unidades de la Defensa en las zonas devastadas por el terremoto y tsunami en el Maule, Chile. Instancia en que la seguridad social se vio comprometida por el aumento explosivo de la delincuencia, sobrepasando ampliamente las capacidades de la fuerza pública hasta llegar al descontrol social, siendo un ejemplo de ello los saqueos de locales comerciales (Bustamante, 2010).

Por otra lado, las capacidades polivalentes de las Fuerzas Armadas, independiente de su rol principal, basadas en la vasta experiencia en operaciones de paz, en el apoyo humanitario, su participación en operaciones con fuerzas multinacionales, entre otras actividades de trabajo conjunto para enfrentar las consecuencias de un determinado evento de gran magnitud (MDN, 2018), han permitido que interactúe y opere con celeridad y eficacia en la fase de respuesta y en apoyar las tareas de ayuda humanitaria durante las siguientes fases del CGRD. Esto hace de la Defensa un recurso importante a la hora de reaccionar antes, durante y después de las consecuencias de una emergencia o desastre, permitiendo fortalecer la gobernanza entre las instituciones responsables y el SNGRD.

Actualmente, la Defensa tiene un rol más activo en el CGRD, su integración está contemplada en los CPC y, ante un evento, en los COE comunales, regionales y nacional. Coordinaciones cívicomilitares que fortalecen el SNGRD y más aún cuando la autoridad política del Ministerio de Defensa promueve la implementación de un Sistema de Gestión de Riesgo y Desastres (MDN, 2019b), estableciendo de esta manera el apoyo irrestricto a la población y al SNPC en las diferentes fases del ciclo del riesgo. 
Es así como la Armada de Chile provee con información técnica que permite fortalecer al sistema de información del SNPC derivándola a la ONEMI y cuya responsabilidad institucional recae en el Servicio Hidrográfico y Oceanográfico de la Armada (SHOA), disponiendo del Sistema Integrado de Predicción y Alarma de Tsunami (SIPAT) que establece una división del país en 21 bloques, estableciendo la evacuación de la o las zonas comprometidas (Armada, 2016) y, el Sistema Nacional de Alarma de Maremotos (SNAM), dotadas de boyas DART (Deep Ocean Assesment and Reporting of Tsunami, por sus siglas en inglés) ubicadas en el océano Pacífico permitiendo monitorear constantemente el período de onda de la costa chilena, considerando que la longitud de onda de un tsunami es evidentemente mayor (SNAM Chile, 2020).

En el caso del Ejército de Chile, el apoyo en esta línea es a través del Instituto Geográfico Militar (IGM) y su área de dominio es la geocartografía del territorio nacional (IGM, 2020), destacando la implementación del Sistema Integrado de Información para Emergencias (SIIE), que permite graficar con mayor claridad las probables áreas de riesgo para la población (Bio Bio Chile, 2013).

Dentro del contexto planteado y en el caso de la Fuerza Aérea de Chile, uno de los apoyos técnicos es a través de la Dirección General de Aeronáutica Civil (DGAC), que además de informar las condiciones meteorológicas locales a nivel nacional, se encuentra correlacionado con la Oficina Nacional de Emergencias (ONEMI, 2016): su objetivo es reaccionar ante las emergencias aeronáuticas civiles. Enlaces e información que fortalecen las capacidades del Centro de Alerta Temprana (CAT) y, por ende, la respuesta del sistema nacional de emergencias.

Cabe además señalar que otros de los apoyos que han realizado las instituciones de la Defensa fuera de las acciones propias desarrolladas dentro de las fases y etapas del CGRD, son las actividades de apoyo a la sociedad civil, empleando los medios de acuerdo a las capacidades de cada una de las ramas castrenses y, en el caso de la Armada de Chile se destacan dos: las propias atingentes al rescate marítimo, emergencias que predominan en verano (Armada, 2018); y a las vinculadas a la fundación Acrux (ACRUX, 2019), cuyo objetivo se centra en realizar operativos médicos de emergencia a lo largo del territorio nacional, abarcando las zonas más aisladas o aquellas carentes de profesionales especialistas. En esto se emplean medios de la institución dispuestos por el Ministerio de Defensa en apoyo al Ministerio de Salud y a la fundación mencionada, trabajo mancomunado que permite disminuir las listas de espera del sistema de atención médica del área pública.

Por su parte, el Ejército de Chile ha desplegado su Hospital Modular de Campaña en diferentes ocasiones, siendo uno de ellos el apoyo al Hospital San José en Santiago, durante la campaña de invierno 2019 y que fue dirigida por el Ministerio de Salud (COSALE, 2019).

En el caso de la Fuerza Aérea de Chile, se destaca el apoyo a la ciudadanía a través del Servicio de Búsqueda y Salvamento Aéreo (SAR, por sus siglas en inglés). Tal organización se presta a cumplir 70 años de servicio a la sociedad (FACH, 2020), disponiendo de transporte aéreo para el traslado tanto de pacientes como de órganos dentro del territorio nacional (Cooperativa, 2019), vital a la hora de colaborar en el proceso para salvar vidas que dependen de una respuesta rápida, y de los medios que en el sistema privado chileno son de un costo elevado o inalcanzables para el paciente.

Complementando lo expuesto anteriormente a la contingencia actual, se destaca el apoyo que las tres ramas de la Defensa mantienen con las diferentes instituciones del Estado en la lucha contra la pandemia global del COVID-19 en Chile. Tales apoyos se articulan dentro del Plan de acción Coronavirus COVID-19 del Ministerio de Salud de Chile (MINSAL, 2020). En el plan, convergen las diferentes unidades médicas de la Defensa en el Estado Mayor Conjunto que, dotado de una mejor coordinación, reforzará el sistema público de salud en tiempos de crisis.

De acuerdo a lo desarrollado, se puede concluir que, al emplear las capacidades polivalentes de las ramas de la Defensa, sumada a las altas capacidades de especialización de los profesionales y al aporte de la sociedad civil organizada, los efectos positivos en el SNGRD son destacables.

\section{EXPERIENCIAS EN ESTADO DE EXCEPCIÓN CONSTITUCIONAL}

Una de las atribuciones especiales del Presidente de la República de Chile es declarar Estado de Excepción Constitucional de acuerdo a lo que establece la normativa legal (BCN, 2005). En este mecanismo de respuesta gubernamental frente a las consecuencias de los eventos de gran magnitud se destacan dos medidas: la primera es la declaración del 'estado de catástrofe', que 
considera "subsanar los efectos de la calamidad pública" (BCN, 1985); y la de 'emergencia', la que incorpora la designación del Jefe de la Defensa Nacional (JDN). El o la JDN será responsable de la jurisdicción afectada de acuerdo a lo que determine la máxima autoridad del país. Estas medidas establecen el empleo de las instituciones de la Defensa para garantizar la seguridad nacional y el apoyo permanente a los diferentes actores del SNPC.

Durante los últimos años, el empleo de las Fuerzas Armadas para fortalecer las condiciones de los organismos que intervienen en la fase de respuesta ante una emergencia o desastres ha aumentado gradualmente. Las principales razones pueden estar relacionadas a sus medios (personal y material), y así como su disposición (legal y de principios) para reaccionar durante crisis y emergencias durante las 24 horas del día, los 7 días de la semana y durante todo el año. Tal polivalencia, permite un mayor grado de maniobrabilidad y eficacia del SNPC.

Entonces, una vez declarado el Estado de Excepción Constitucional por el Presidente de la República, éste delega algunas de sus facultades al Jefe de la Defensa Nacional, autoridad militar que asume el control de la zona afectada en apoyo al sistema de seguridad interior, disponiendo las acciones necesarias para garantizar una respuesta oportuna y eficaz a las víctimas (BCN, 1985).

Dentro del contexto descrito previamente y con el objetivo de tener una perspectiva más clara de la importancia que ha tenido el empleo de las ramas de la Defensa en las emergencias, a modo de ejemplo, se menciona el incendio que afectó la comuna de Valparaíso, Chile, en enero del año 2017 (Diario U de Chile, 2017). En esas fechas, el gobierno de entonces declara Estado de Excepción Constitucional, destacándose las experiencias de dos oficiales de la Fuerzas Armadas en el desarrollo de los acontecimientos: el Jefe de la Defensa Nacional y el Comandante del Grupo Operativo de Mantenimiento del Orden Público (Cabezas, 2017). Experiencias que han permitido observar las condiciones de conducción en un estado tan complejo como lo es un desastre, tanto por la cantidad de afectados, como de la superficie geográfica comprometida, o por la multiplicidad de actores involucrados, develando un panorama adverso para cualquier tipo de conducción, ya sea esta civil o militar.

Dicho lo anterior, el Oficial General' designado como JDN destacó que durante su interacción político-militar con las autoridades de gobierno interior y las propias del COE fueron satisfactorias debido a que, regionalmente, tienen bien definido sus obligaciones, atribuciones y responsabilidades, por lo tanto, las gestiones para superar la emergencia fueron más eficientes y eficaces. Además, indica que durante las primeras horas de la emergencia es donde fluye mucha información y a través de ellas, se determinaron las prioridades de acción y se asignaron las tareas para posteriormente reevaluarlas, permitiendo determinar el nivel de progreso de ellas y así, en caso de ser necesario, reorganizar los esfuerzos hacia las áreas que necesitan más apoyo o que por las circunstancias propias de la emergencia, surgen nuevas necesidades que requieren ser atendidas. También señala que la máxima prioridad en estas condiciones es siempre salvaguardar la vida humana, la seguridad del personal de apoyo como bomberos y personal sanitario, la protección de bienes u otras tareas necesarias para restaurar los servicios de primera necesidad y así superar la emergencia en el menor tiempo posible. Mencionó, además, que se tiene la errónea percepción que los damnificados son reticentes a la presencia de las Fuerzas Armadas en el área afectada por un evento destructivo. Por el contrario, su experiencia es que ellos reciben muy bien al personal de la Defensa debido a que aumenta la sensación de seguridad, tranquilidad (El Mercurio de Valparaíso, 2017) y confianza en que las acciones tomadas van en directa relación a superar la emergencia, ello complementado a los resultados que demuestran claramente que, al mejorar las condiciones del orden público en conjunto con las policías, reducen la actividad delictual significativamente.

A su vez, cumplidos los plazos establecidos por el decreto que determinó tal acción, el JDN y la autoridad política regional desarrollan una estrategia de retiro de las fuerzas desplegadas, con el objetivo de que la autoridad civil respectiva retome el control, no sin antes presentar los informes e indicadores basados en el cumplimiento de los objetivos planteados para reestablecer el orden público y el apoyo entregado en el proceso humanitario, o sea, desarrollado dentro de sus facultades para garantizar la seguridad interior de la zona afectada.

Por otra parte y bajo la condición jerárquica del oficial general mencionado, se encuentra el Grupo Operativo de Mantenimiento del Orden Público, compuesto por personal militar de las tres ramas de la Defensa de la zona comprometida o jurisdiccional, y cuyo Oficial Jefe ${ }^{2}$ al mando menciona que: una de las primeras tareas que realizó el Grupo en la zona de despliegue, fue un estudio de seguridad de toda el área afectada para determinar los niveles de riesgos ya sean

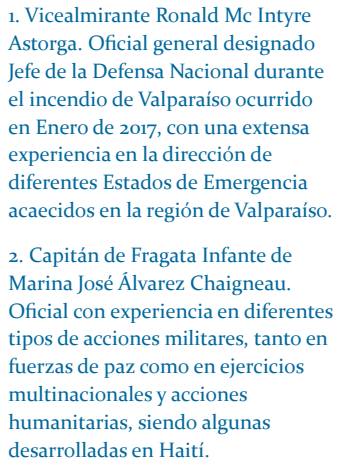


estos de vulnerabilidad social, delictuales o geográficos. Estos últimos producto de las variaciones que sufre el entorno sobre todo ante un sismo o un incendio de grandes proporciones, estudios o informes que son actualizados constantemente durante toda la permanencia.

En relación a la seguridad pública, el grupo mencionado asume el resguardo de la totalidad del área jurisdiccional asignada al Estado de Excepción Constitucional, tomando acciones de carácter preventivo y contando siempre con el apoyo policial cuando estos eran requeridos. Trabajo en equipo que restauró el orden público, la tranquilidad a la población y a los grupos de apoyo civil, resguardando a su vez centros de acopio, centros de control médico, de apoyo social, entre otros recintos necesarios para las operaciones tanto civiles como militares.

Considerando el carácter de trabajo preventivo de las fuerzas desplegadas, es importante aclarar que éstas no llevan a cabo tareas investigativas de un acto punitivo o legal, siendo labores o facultades exclusivamente policíacas, pero si, la respectiva denuncia ante un hecho constitutivo de delito, de ahí la importancia del trabajo mancomunado entre las diferentes instituciones, en este caso, militares y policiales.

Por otra parte, cabe recordar que el entrenamiento militar que reciben y realizan las Fuerzas Armadas a nivel nacional es y será para desarrollar operaciones bélicas en resguardo de la soberanía de la nación basadas en los "principios de la política de Defensa" (MDN, 2017, p.96). Sin embargo, existe un entrenamiento complementario para las misiones u operaciones de paz que se desarrollan bajo lo establecido por la Organización de las Naciones Unidas (ONU), entrenamiento que se realiza en el 'Centro Conjunto para Operaciones de Paz de Chile' (CECOPAC) cuya misión es "avocarse a la docencia, instrucción y preparación de personal destinado a participar en Operaciones de Paz, mediante el desarrollo de cursos, así como al estudio, investigación y asesoría en tales materias a diferentes estamentos" (CECOPAC, 2002). El CECOPAC se encarga de instruir al personal de las diferentes instituciones sean estos civiles, militares y policiales que se encuentran prestas a ser desplegadas para cumplir una determinada misión en el marco de las Naciones Unidas (UN, por sus siglas en inglés). Cursos que son impartidos a través de módulos que contemplan las materias obligatorias basadas en los estándares de las operaciones de paz, como así también lo relacionado a la información del área de misión o de despliegue y una fase práctica objeto emplear en terreno los conocimientos adquiridos. Competencias técnicas que, complementado al entrenamiento militar, las experiencias y el trabajo realizado en las diferentes misiones de paz como la de Haití, han permitido diversificar aún más las funciones de las unidades militares, y de hacerlas más polivalentes en las operaciones humanitarias. Por lo expuesto anteriormente, éstas pueden ser efectivamente empleadas para la seguridad interior en todas las fases del CGRD.

De acuerdo a lo expresado, las capacidades profesionales, la experiencia y el conocimiento empírico, son capacidades que promueven una participación eficaz y eficiente de las Fuerzas Armadas durante un desastre, sobre todo ante una contingencia donde son relevantes las ventajas de mando y control frente a situaciones de caos y desborde del orden público.

\section{EXPERIENCIAS EN EMERGENCIAS MINERAS}

A nivel nacional, otra de las experiencias de apoyo de la Defensa fuera del contexto regular ocurrió en Copiapó, Chile, en agosto de 2010, precisamente en la mina San José. Un derrumbe bloqueó el acceso dejando a 33 mineros atrapados en su interior, consecuencia que generó una emergencia que conmovió al mundo conocida como 'los 33'. Circunstancias donde el Ejército y la Armada de Chile también estuvieron apoyando con funcionarios durante el proceso de planificación y el rescate. El Ejército contribuyó en el aspecto logístico, mientras que la Armada asesoró al gobierno en materias técnicas referidas a la condición de profundidad en que se encontraban los 33 mineros, requiriendo la presencia de personal especialista en sumersión y del área médica para determinar las acciones o medidas a tomar bajo las condiciones de confinamiento extremo en que se encontraban los trabajadores. Paralelamente, la empresa Astilleros y Maestranzas de la Armada (ASMAR) desarrolló un proyecto que permitiría al más breve plazo la construcción de un artefacto (Plubimetro, 2010) que asegure la extracción de los mineros atrapados a través del conducto hecho por una de las perforadoras. Así mismo, durante el proceso de planificación del rescate se realizó una capacitación al equipo de salud civil desplegado en el campamento de rescate. Haciendo referencia a las técnicas y tecnologías de la medicina de combate utilizadas en las unidades de la Infantería de Marina, el curso fue titulado Manejo Inicial del Trauma Estación 33 (MITE33), e impartido por el personal institucional ${ }^{3}$ y dos profesionales civiles especialistas en trauma y rescate de la salud prehospitalaria ${ }^{4}$. El entrenamiento se centró en la evaluación y
3. Capitán de Fragata Sanidad Naval Dr. Andrés Llarena Astudillo y Sargento Infante de Marina Enfermero Gabriel Cabezas Ríos.

4. Dra. Ximena Grove Maureira, médico especialista en trauma y atención prehospitalaria, Mg. en Gestión de Riesgo de Desastres con mención en Políticas Publicas y, Robinson Talavera Cerda, Enfermero Universitario, Bombero especialista en rescate, Mg. en Gestión de Riesgo de Desastres con mención en Ingeniería Logística. 
tratamiento inmediato de un paciente crítico en condiciones extremas o, como lo era en este caso, estar preparados ante una eventualidad durante los trabajos que debían realizar los mineros a 700 metros de profundidad una vez iniciado el ciclo de rompimiento de la perforadora. Tales riesgos debían ser considerados debido a la exposición física y mental a la que serían expuesto los mineros, teniendo presente además que dicha área de trabajo era el único sector establecido para la extracción.

El resultado positivo del rescate es conocido por todo el mundo, pero lo relevante para este estudio es que las instituciones y organizaciones públicas y privadas que participaron, y en particular la Defensa, se articularon de manera eficaz para cumplir un objetivo común, la extracción con vida de los 33 mineros de la Mina San José de Copiapó. Cada aporte fue necesario para cumplir el objetivo mencionado, demostrando además que las instituciones militares pueden contribuir con medios, personal instruido y profesional para desarrollar tareas que en ocasiones son específicas y muy diferentes a su rol principal.

Otra de las experiencias de apoyo de la Defensa en emergencias mineras fue la ocurrida el 25 de enero del año 2011 en la mina La Preciosa ubicada en Sardinata, Colombia. Una explosión en interior de la mina dejo 21 víctimas fatales, ante lo cual el Gobierno de Chile decide enviar un grupo de expertos para apoyar en las labores de rescate e investigación. El equipo fue constituido por dos funcionarios de la Armada de Chile, un funcionario de Carabineros de Chile y un profesional de la Corporación Nacional del Cobre de Chile (CODELCO). Una vez en Colombia, el equipo se integró a la comisión investigadora (La Tercera, 2011) para posteriormente ser trasladados a la mina (Armada, 2011). Junto al resto del equipo de profesionales Colombianos, iniciaron las primeras indagaciones para establecer las causas de la tragedia y así prevenir o mitigar los riesgos a futuro.

En base a las experiencias descritas previamente y teniendo presente que los escenarios de desempeño son alejados del rol principal de las Fuerzas Armadas, antes explicado, se puede concluir que las capacidades profesionales, tecnológicas y los medios con que cuenta la Defensa, apuntan a ser una buena alternativa o herramienta para fortalecer el SNGRD, especialmente en la fase de respuesta tanto dentro como fuera de Chile.

\section{CONCLUSIONES}

Existe cierta lógica al creer que las consecuencias negativas de un evento de gran magnitud o el nivel de destrucción posee una relación con el nivel de desarrollo de cada país, circunstancias que podrían llegar a comprometer no tan solo el orden social, sino también las capacidades de sustento y de desarrollo. Por consiguiente, en el contexto chileno es relevante que se deban establecer políticas públicas que permitan enfrentar de mejor forma los eventos extremos y desastres, promoviendo el perfeccionamiento de las capacidades de respuesta del Sistema Nacional de Protección Civil (SNPC) en Chile. Por otro lado, las diferentes experiencias de desastres han permitido avanzar en estrategias para desarrollar y potenciar la gobernanza y, en su sentido lógico, avanzar en los procesos de Gestión del Riesgo de Desastre (GRD), fortaleciendo el apoyo y aprendizaje recíproco a nivel nacional e internacional, mejorando en forma constante la estructura gubernamental en todos sus niveles. De este proceso de desarrollo, las Fuerzas Armadas se han mostrado positivamente como un instrumento operativo de seguridad interior en la fase de respuesta, y con ello un elemento importante a considerar en el SNPC y la GRD.

En el aspecto internacional, la interacción de las autoridades de la Defensa y de las fuerzas operativas en los diferentes ejercicios multinacionales, han permitido fortalecer las condiciones estratégicas, la integración, el trabajo conjunto, la reciprocidad de procedimientos y las experiencias de las diferentes organizaciones que confluyen en el ciclo de la Gestión del Riesgo de Desastre (CGRD), tanto en Chile como en la región.

Por su parte, la modernización del actual SNPC ha permitido el fortalecimiento de la red de los sistemas de emergencias, con una institucionalidad provista de atribuciones legales necesarias para cumplir con una política pública capaz de hacer frente al escenario de riesgo nacional y a su vez, consolidar el plan estratégico de la gestión del riesgo de desastres. La presencia de las Fuerzas Armadas en todo el territorio nacional y su polivalencia, han contribuido también a la descentralización operativa del actual SNPC, en materia de respuesta. Tal instrumentalización ha fortalecido no tan solo al Ministerio del Interior, sino también a otros ministerios: sobre todo cuando estos últimos han requerido medios y recurso humano adicional ante circunstancias adversas que 
pueden generan un riesgo para la sociedad. Además, la Defensa pone a disposición de la ONEMI la información técnica necesaria para la toma de decisiones, y apoyando iniciativas gubernamentales donde las capacidades militares permiten establecer la conectividad de la población más aislada ante una determinada necesidad social.

Todas las acciones que involucran un trabajo conjunto requieren necesariamente de una conducción estratégica capaz de hacer interactuar mancomunadamente a todos los actores que intervienen tanto en la respuesta como en las demás fases del CGRD. Escenarios que difieren uno de otro, o son poco convencionales, como el apoyo realizado a las emergencias mineras o las acciones cívicas de salud desarrolladas en el país. Instituciones militares que, independiente de las circunstancias, contribuyen en forma conjunta a mejorar las condiciones de apoyo en cada nivel de respuesta que van en directo beneficio de la sociedad.

Finalmente, sabemos que los recursos siempre serán limitados ante las consecuencias de un evento destructivo o de gran magnitud, es por ello la relevancia de contar con una organización de emergencia o sistema de protección civil multidisciplinaria, con personal y medios dispuestos en todo el territorio nacional las 24 horas del día, haciendo que la gestión gubernamental sea más eficiente para el Estado. La respuesta a emergencias y desastres no serían posible, o las dificultades serían mayores, si no se contara con profesionales cuyas capacidades y experiencia en escenarios adversos elevan el nivel de eficacia de los sistemas de mando y control. Por lo anterior, los funcionarios de la Defensa han sido un aporte relevante debido a su continua preparación para superar adversidades o situaciones propias de una carrera militar condicionada a su rol principal, pero también una preparación que contribuye permanentemente al fortalecimiento del SNGRD en todas las fases y etapas del ciclo de gestión del riesgo de desastre.

\section{REFERENCIAS}

ACRUX. (2019). Ministro de Defensa inaugura operativo médico número 10o de Fundación Acrux a bordo del Buque Sargento Aldea en Iquique. Fundación Acrux. Recuperado de: https:// fundacionacrux.cl/ministro-de-defensa-inaugura-operativo-medico-numero-10o-de-fundacionacrux-a-bordo-del-buque-sargento-aldea/

Armada de Chile. (2011). Avances en las labores de los rescatistas chilenos en mina "La Preciosa" de Colombia. Armada.cl. Recuperado de: https://www.armada.cl/armada/site/cache/imprimir/pags/ imprimir15952408567654.html

Armada de Chile. (2016). Nuevo sistema del SHOA permitirá segmentar las evacuaciones en caso de amenaza de tsunami. Armada.cl. Recuperado de: https://www.armada.cl/armada/noticiasnavales/nuevo-sistema-del-shoa-permitira-segmentar-las-evacuaciones-en-caso-de-amenaza-detsunami/2016-06-10/143627.html

Armada de Chile. (2018). Rescates Marítimos. Armada.cl. Recuperado de: https://www.armada.cl/ armada/rescates-maritimos/2018-08-10/120442.html

BCN. (1985). Ley Orgánica Constitucional de los Estados de Excepción. Biblioteca del Congreso Nacional

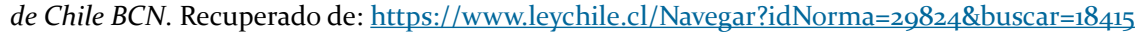

BCN. (2002). Decreto 254 Acuerdo con Argentina sobre cooperación en materia de Catástrofes. Biblioteca del Congreso Nacional de Chile BCN. Recuperado de: https://www.leychile.cl/ Navegar?idNorma $=207090$

BCN. (2005). Constitución Política de la República de Chile. Biblioteca del Congreso Nacional de Chile $B C N$. Recuperado de: $\underline{\text { https://www.leychile.cl/Navegar?idNorma }=242302}$

BCN. (2010). Estatuto Orgánico del Ministerio de Defensa Nacional. Biblioteca del Congreso Nacional de Chile BCN. Recuperado de: https://www.leychile.cl/Navegar?idNorma $=1010682 \&$ buscar $=20424$

BCN. (2011). Modifica el Decreto $N^{\circ} 156$ de 2002 y determina constitución de los Comités de Operaciones de Emergencia. Biblioteca del Congreso Nacional de Chile BCN. Recuperado de: https:// www.leychile.cl/Navegar?idNorma $=1023837$

BCN. (2015). Texto refundido, coordinado y sistematizado de la Constitución Política de la República de Chile. Biblioteca del Congreso Nacional de Chile BCN. Recuperado de: https://www.leychile.cl/ Navegar?idNorma $=242302$

BCN. (2017). Aprueba Plan Nacional de Emergencia. Biblioteca del Congreso Nacional de Chile BCN. 
Recuperado de: $\underline{\text { https: } / / w w w . l e y c h i l e . c l / N a v e g a r ? i d N o r m a=1106167 \& b u s c a r=d t o+1434+i n t e r i o r ~}$

Bio Bio Chile. (15 de junio, 2013). Onemi firma convenio con Instituto Geográfico Militar. BioBioChile. cl. Recuperado de: https://www.biobiochile.cl/noticias/2013/o6/15/onemi-firma-convenio-coninstituto-geografico-militar.shtml

Bustamante, R. (2010, 28 de febrero). Terremoto en Chile: rescates y saqueos en Concepción. (R. Bustamante, Recopilador). BBC Mundo. Recuperado de https://www.bbc.com/mundo/america latina/2010/02/100228 1623 chile saqueos gtg.shtml

Cabezas, G. (2017). El Estado, El Gobierno, La Defensa y el Sistema Nacional de Protección Civil en los Eventos Destructivos en Chile. Tesis de Magister, Universidad de Valparaíso. Recuperado de: http:// catalogobibliotecas.uv.cl/cgi-bin/koha/opac-detail.pl?biblionumber=106237

CECOPAC. (2002). Historia. Centro Conjunto de Operaciones de Paz de Chile CECOPAC. Recuperado de: http://www.cecopac.cl/?page id=199

CMDA. (2018a). Conferencia de Ministros de Defensa de las Américas. CMDA. Recuperado de: https:// www.cmda-info.net/

CMDA. (2018b). Anexo Recomendaciones de los Cinco Grupos de Trabajos Ad-Hoc. CMDA. Recuperado de: https://docs.wixstatic.com/ugd/c3ec19 2a88bd5d58334093bc74ecoec1210dd4.pdf

CNID. (2016). Hacia un Chile resiliente frente a desastres: Una oportunidad. Santiago: CREDEN.

Cooperativa. (o4 de agosto, 2019). FACH traslada a Santiago órganos donados de joven muerto en accidente en Copiapó. Cooperativa.cl Recuperado de: https://www.cooperativa.cl/noticias/pais/ region-de-atacama/fach-traslada-a-santiago-organos-donados-de-joven-muerto-en-accidenteen/2019-08-04/181729.html

COSALE. (2019). Culmina apoyo del HMCE al Hospital San José por campaña de invierno. Comando de Salud del Ejército de Chile COSALE. Recuperado de: http://www.cosale.cl/ culmina-apoyo-del-hmce-al-hospital-san-jose-por-campana-de-invierno/

Diario U. de Chile. (2017, 02 de enero). Gran incendio afecta a cerros de Valparaíso. Radio U. de Chile. Recuperado de: https://radio.uchile.cl/2017/01/o2/gran-incendio-afecta-a-cerros-de-valparaiso/

EMCO. (2014). Dirección Operaciones y Conducción Conjunta. Estado Mayor Conjunto de Chile EMCO. Recuperado de: https://www.emco.mil.cl/?page id=255.

EL Mercurio de Valparaíso. (2017, 31 de enero). Gobierno levanta Estado de Excepción en zonas afectadas por incendio en Playa Ancha. EL Mercurio de Valparaíso. Recuperado de: http://www. mercuriovalpo.cl/impresa/2017/01/31/full/cuerpo-principal/5/

FACH. (2020). Servicio de Búsqueda y Salvamento Aéreo. Fuerza Aérea de Chile FACH. Recuperado de: https://www.fach.mil.cl/sar/quienes\%20somos.htm

IGM. (2020). Misión y Visión. Instituto Geográfico Militar IGM. Recuperado de: https://www.igm.cl/ index.php

La Tercera. (2011, 26 de enero). Brigada de rescatistas chilenos parte a Colombia a labores de salvataje por tragedia en mina. La Tercera. Recuperado de: https://www.latercera.com/noticia/ brigada-de-rescatistas-chilenos-parte-a-colombia-a-labores-de-salvataje-por-tragedia-en-mina/

Ministerio de Defensa Nacional MDN. (2017). Libro de la Defensa Nacional. Santiago: Gráfica Marmor. Recuperado de: https://www.defensa.cl/media/LibroDefensa.pdf

Ministerio de Defensa Nacional MDN. (2018). Ministro Espina en ejercicio “Solidaridad 2018”: “La relación entre las Fuerzas Armadas de Chile y Argentina son óptimas". Defensa.cl. Recuperado de: https://www.defensa.cl/noticias/ministro-espina-en-ejercicio-solidaridad-2018-la-relacion-entrelas-fuerzas-armadas-de-chile-y-argen/

Ministerio de Defensa Nacional MDN. (2019a). IV Reunión de Ministros de Defensa del Pacífico Sur. Defensa.cl. Recuperado de: https://www.defensa.cl/noticias/ministro-de-defensa-nacionalparticipa-en-la-iv-reunion-de-ministros-de-defensa-del-pacifico-sur-spd/

Ministerio de Defensa Nacional MDN. (2019b). Mensaje Presidencial. Defensa.cl. Recuperado de: https://www.defensa.cl/wp-content/uploads/Mensaje_Presidencial_Defensa.pdf

Ministerio del Salud MINSAL. (2020). Ministros de Salud y Defensa se coordinan para disponer de hospitales de las Fuerzas Armadas para reforzar red asistencial por COVID-19. Minsal.cl. Recuperado de: https://www.minsal.cl/ministros-de-salud-y-defensa-se-coordinan-para-disponer-de-hospitalesde-las-fuerzas-armadas-para-reforzar-red-asistencial-por-covid-19/ 
Oficina Nacional de Emergencia ONEMI. (2002). Plan Nacional de Emergencia. Santiago: Ministerio del Interior. Recuperado de: http://repositoriodigitalonemi.cl/web/bitstream/handle/2012/1683/DTO156 13-JUN-2002.pdf? sequence $=4$

ONEMI. (2016). ONEMI y DGAC firmaron Acuerdo de Cooperación en materias de emergencias aéreas en aeropuertos y aeródromos. Onemi.cl. Recuperado de: https://www.onemi.gov.cl/noticia/onemiy-dgac-firmaron-acuerdo-de-cooperacion-en-materias-de-emergencias-aereas-en-aeropuertos-yaerodromos/

ONEMI. (2018). Plan Estratégico 2019 - 2023. Santiago: ONEMI.

ONEMI. (2019). Declaración de Chile en la VI Plataforma Global. Santiago: ONEMI. Recuperado de: https://www.onemi.gov.cl/wp-content/uploads/2019/05/Declaracion-Chilena_PG19-Rev-ONEMIMINREL.pdf

Organización de Naciones Unidas ONU. (2015). Marco para Reducción del Riesgo de Desastre de 2015-2030. Sendai, Japón: Naciones Unidas. Recuperado de: https://www.unisdr.org/files/43291 spanishsendaiframeworkfordisasterri.pdf

Plubimetro. (2010, 13 de septiembre). Así será la cápsula que diseñó Asmar para el rescate de los mineros. Publimetro.cl. Recuperado de: https://www.publimetro.cl/cl/nacional/2010/og/13/asi-seracapsula-que-diseno-asmar-rescate-mineros.html

Embajada de los EE. UU. en Chile. (2014). Partnership of the Americas. Embajada de los EE. UU. en Chile. Recuperado de: https://cl.usembassy.gov/es/united-states-marine-corps-participatehumanitarian-disaster-relief-exercise-partnership-americas-2014-es/

Senado de Chile. (2019). Estado de tramitación proyecto de ley. Senado.cl. Recuperado de: http://www. senado.cl/appsenado/templates/tramitacion/index.php?boletin ini=7550-06

Servicio Nacional de Geología y Minería SERNAGEOMIN. (2020). Sernageomin da a conocer nuevo ranking de volcanes. Sernageomin.cl. Recuperado de: https://www.sernageomin.cl/ sernageomin-da-a-conocer-nuevo-ranking-de-volcanes/

SNAM Chile. (2020). Sistema Nacional de Alarma de Maremotos - SNAM. SNAM Chile. Recuperado de: http://www.snamchile.cl/index.php?p=snam\&enl=3

Oficina de las Naciones Unidas para la Reducción del Riesgo de Desastres UNISDR. (2019). Plataforma Global de Reducción del Riesgo de Desastres. UNISDR.org. Recuperado de: https://www.unisdr.org/ conference/2019/globalplatform/home 